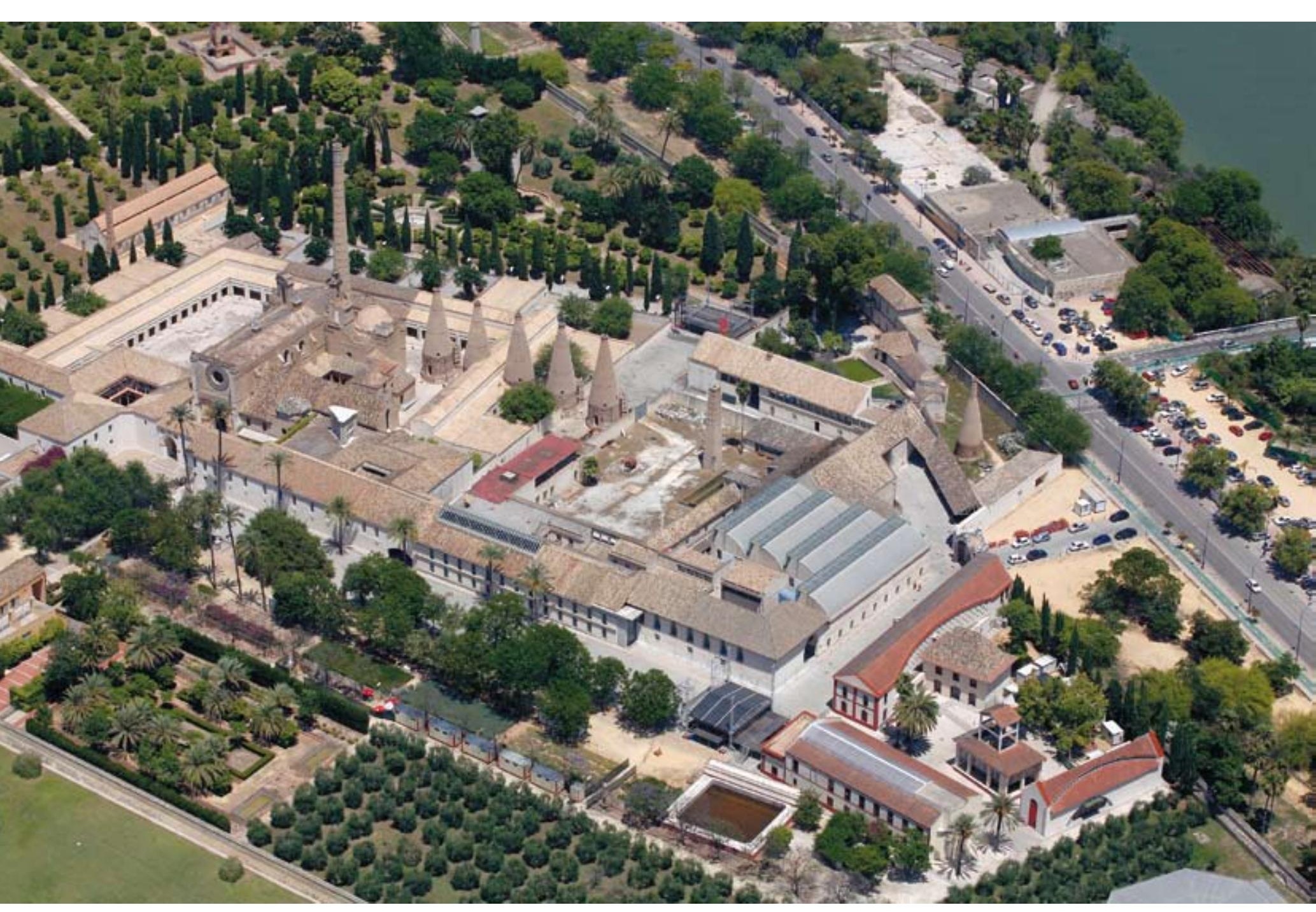

Vista aérea del antiguo Monasterio de la Cartuja de Santa María de Las Cuevas, situado en la Isla de la Cartuja. Sevilla. Foto: Carlos Jiménez. Fuente: Fondo Gráfico IAPH 


\section{El Servicio de Cartografía Digital del Instituto Andaluz del Patrimonio Histórico}

José Manuel Díaz Iglesias, Centro de Documentación y Estudios del IAPH

\section{Resumen}

A lo largo de los últimos veinte años, en el Centro de Documentación y Estudios del Instituto Andaluz del Patrimonio Histórico (IAPH) se han emprendido diversas iniciativas para contribuir al estudio y conocimiento del patrimonio cultural andaluz. La transferencia de la información generada ha estado siempre entre los objetivos básicos de esta Institución, para lo cual a lo largo de estas dos décadas ha desarrollado diferentes líneas de actuación.

La difusión de la información geográfica de las entidades patrimoniales de Andalucia forma parte de estas acciones. En este sentido, desde el IAPH se han abordado dos iniciativas centradas en el desarrollo de un producto de difusión y la prestación de un servicio especializado de acceso a la información espacial.

En este artículo se describe y analiza el Servicio de Cartografía Digital del IAPH en el contexto de la legislación y planificación en materia de difusión de la información geográfica, así como en el marco de la gestión de los servicios públicos de la administración andaluza.

\section{Palabras clave}

Andalucia / Bases de datos / Cartografía digital / Centro de Documentación y Estudios / Difusión / Información geográfica / Instituto Andaluz del Patrimonio Histórico / Metodología / Patrimonio cultural / Servicio de cartografía digital / Tecnologías de la información geográfica / Transferencia de la información 


\section{SERVICIOS Y PRODUCTOS DE INFORMACIÓN}

Desde su creación en 1989, el Instituto Andaluz del Patrimonio Histórico (IAPH) ha realizado diversas actuaciones en el ámbito de la documentación, el estudio y la transferencia de la información del patrimonio cultural andaluz. Desde el Centro de Documentación y Estudios de esta Institución se abordó el desarrollo del Sistema de Información del Patrimonio Histórico de Andalucía (SIPHA) con el objetivo de documentar de forma integrada el conjunto de los datos que identificaban y describian la información del patrimonio histórico andaluz (LADRÓN DE GUEVARA; MUÑOZ, 2007).

A medida que este proyecto avanzaba se comenzó a trabajar en la implementación de diversas estrategias complementarias para la transferencia de esta producción especializada. Con el objetivo de llevar a cabo actuaciones que se adaptaran a las necesidades de diferentes tipos de usuarios se han ido definiendo distintos recursos que, hoy en dia, se materializan en la coexistencia de dos líneas de difusión de la información: los servicios de información bajo demanda y los productos de difusión interactivos o servicios de acceso directo a través del portal web de la Institución.

En cuanto a los primeros, en el año 1995 se crea el Servicio de Información del IAPH (BLANCO; LIMÓN; MADRID, 2007). Con ello se dotaba a la Institución de un área especializada para la atención directa de las demandas de información sobre los bienes culturales, así como para el estudio de las necesidades de los usuarios, la planificación y la coordinación de los servicios.

La evolución de los servicios de información del Instituto Andaluz del Patrimonio Histórico (IAPH) culmina en el año 2005, en el marco del Plan Director de organización para la Calidad de los Servicios de la Junta de Andalucia, con la elaboración y aprobación de la Carta de Servicios del IAPH (BLANCO; ORTEGA, 2005). La finalidad de este documento es informar a los usuarios de los servicios que se gestionan, de las condiciones en las que se prestan y de los compromisos de calidad que se asumen junto a ellos.

En la medida en que Internet se convertía no sólo en un medio privilegiado para la difusión de la información, sino que se afianzaba como el principal canal de comunicación, a la implantación de servicios especializados se sumó el establecimiento de nuevas líneas alternativas a través del desarrollo de productos de información especificos. En este sentido, en 1999 se pone en marcha el servicio de acceso a los datos básicos del patrimonio inmueble arqueológico (SÁNCHEZ; ORTEGA; DÍAZ, 1999). Esta experiencia se hizo extensiva poco después al resto del patrimonio inmueble (MUÑOZ; ORTEGA, 2001). Desde entonces, se ha ido rediseñando este producto y creando otros nuevos, de forma que en la actualidad es posible acceder a una gran variedad de contenidos a través de diferentes recursos.

\section{SERVICIOS DE INFORMACIÓN PATRIMONIAL EN ESPAÑA: LA DIFUSIÓN DE LA INFORMACIÓN GEOGRÁFICA}

Al tiempo que se emprendian en Andalucia las primeras tareas, por parte del IAPH, para la puesta en marcha de servicios de acceso y consulta de la información patrimonial, otros organismos vinculados a la gestión del patrimonio cultural en España desarollaban iniciativas similares. Entre ellos hay que señalar, por un lado, la publicación por parte de la Dirección General de Bellas Artes y Bienes Culturales del Ministerio de Cultura de un servicio de consulta de los datos básicos de los bienes inmuebles inscritos en el Registro de Bienes de Interés Cultural, cuya puesta en marcha era incluso anterior. Y, por otro, la publicación del servicio de consulta del Inventario de Yacimientos Arqueológicos de la Comunidad Valenciana, siendo especialmente reseñable en este servicio la publicación de los datos espaciales que definian su localización.

Si inicialmente estos trabajos estaban centrados en la creación de servicios de acceso a los datos básicos de identificación y descripción de las entidades patrimoniales, ha sido especialmente en los últimos años cuando han comenzado a surgir nuevos recursos para la difusión de la información y, concretamente, para el acceso a la consulta de la información geográfica, existiendo diversos factores que han desencadenado esta eclosión.

Durante la última década, las diferentes administraciones regionales han ido implantando sistemas de información territorial como instrumentos para el desarrollo de sus competencias. En el ámbito de la administración cultural, en la medida en que se gestionan entidades vinculadas al territorio, resulta ineludible la utilización de estos sistemas como herramientas de apoyo para su conocimiento y planificación. Sin embargo, su grado de utilización en España resulta desigual.

En el año 2004 la Comunidad Autónoma de Murcia publica un estudio con el objetivo de conocer el nivel de desarrollo, la estructura y las tendencias de los sistemas de información territorial en España (ESTUDIO, 2004). Aunque en esa fecha aún eran escasas las administraciones culturales que utilizaban las tecnologías de la información geográfica en el marco de un sistema de información, al menos es importante destacar que su empleo ya comenzaba a iniciarse en la administración cultural.

En este contexto, la aprobación de la Directiva comunitaria 2007/2/CE, de 14 de marzo de 2007, para el establecimiento de una infraestructura de información espacial en la Unión Europea (Inspire) ha venido a incentivar esta dinámica. Como respuesta al mandato de esta Directiva, y para garantizar la accesibilidad a la información espacial, han surgido en España múltiples iniciativas centradas en la adopción de los recursos tecnológicos necesarios para la publicación de servicios estándar de visualización e intercambio de información. Es más, el surgimiento y desarrollo de estas propuestas es incluso anterior a la aprobación de esta Direc- 


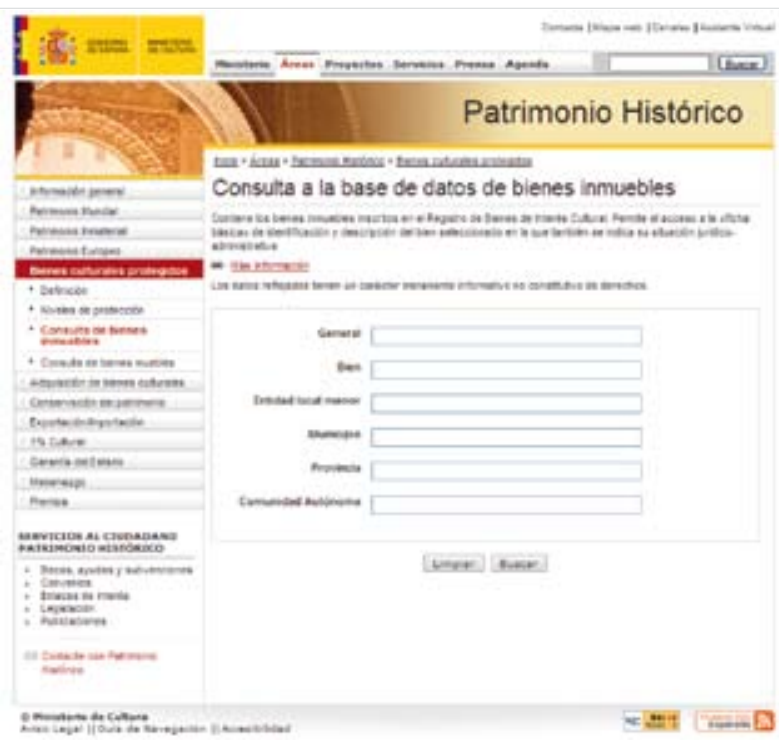

Servicio de consulta de inmuebles inscritos en el Registro de Bienes de Interés Cultural del Ministerio de Cultura: www.mcu.es/bienes/cargarFiltroBienesInmuebles. do? layout=bienesInmuebles\&cache=init\&language $=e s$

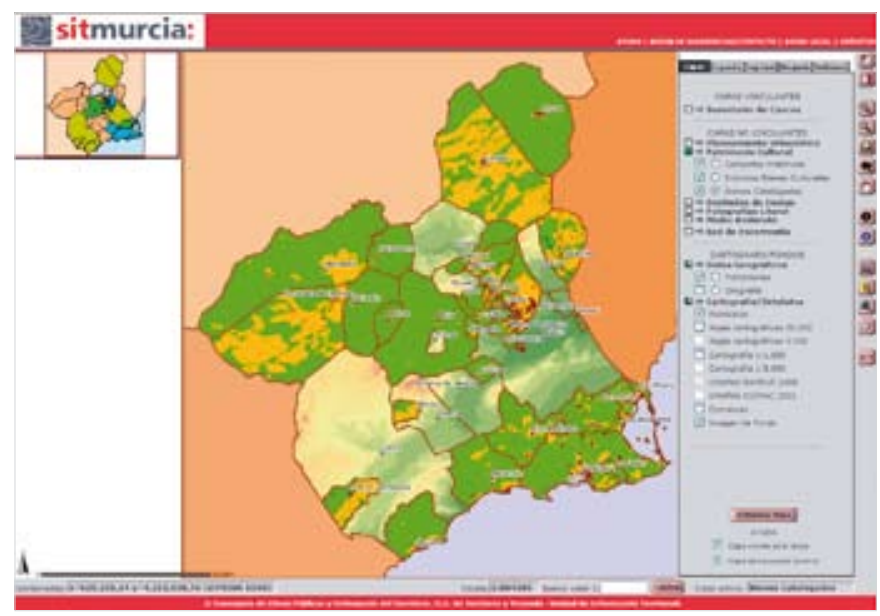

Sistema de Información Territorial de la Región de Murcia: massotti.carm.es/ website/str/viewer

tiva, y en ellas han participado casi de forma generalizada todas las administraciones autonómicas (RODRÍGUEZ et ál., 2007).

La progresiva incorporación de las tecnologias de la información geográfica en las administraciones autonómicas y el seguimiento de las determinaciones de Inspire han propiciado la puesta en valor de la información geográfica, convirtiéndose la difusión y el acceso a la misma en una necesidad demandada desde diversos sectores.

En este sentido, hay que considerar también los contenidos de la Ley 27/2006, de 18 de julio, por la que se regulan los derechos de acceso a la información, de participación pública y de acceso a la justicia en materia de medio ambiente. Esta normativa tiene por objeto fundamental regular los derechos de acceso a la información ambiental que obren en poder de las autoridades públicas, garantizando la difusión y puesta a disposición del público de la información ambiental de manera paulatina y con un grado de amplitud, de sistemática y de tecnología lo más amplio posible.

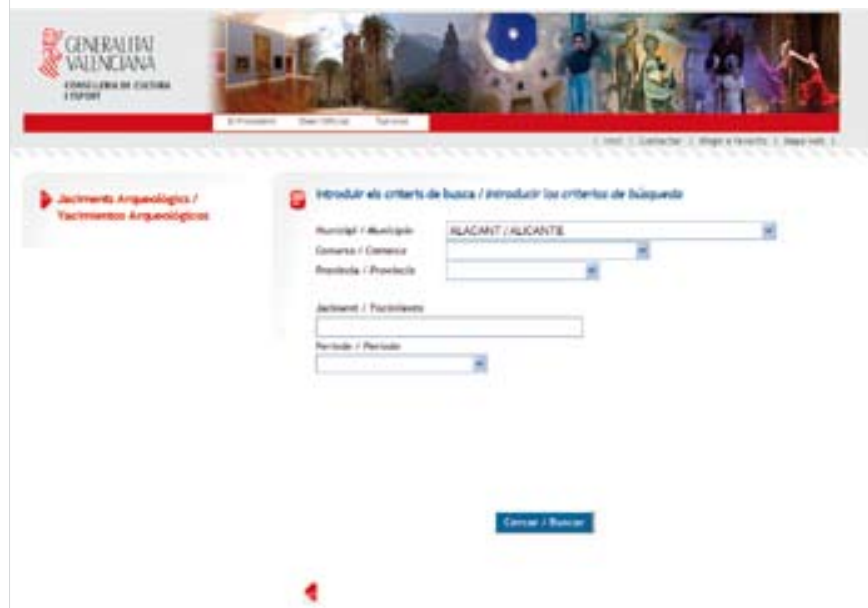

Servicio de consulta del Inventario de Yacimientos Arqueológicos de la comunidad valenciana: www.cult.gva.es/dgpa/yacimientos/consulta.asp

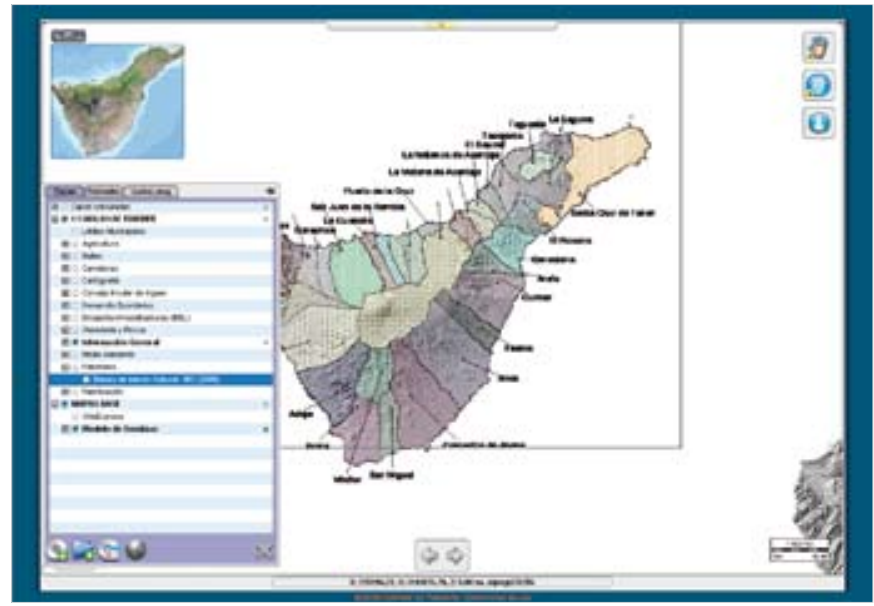

Atlas digital de Tenerife: atlastenerife.es/TeIDE2/Visor.do

En lo referente a la información espacial del patrimonio cultural, Inspire distingue a ésta entre el conjunto de datos espaciales sobre los que han de aplicarse sus determinaciones. En el Anexo I de esta Directiva se mencionan los Lugares Protegidos, entre los cuales se contempla el patrimonio cultural. Del mismo modo, la mencionada Ley 27/2006 considera al Patrimonio Histórico como parte de la información medioambiental.

Como consecuencia, en la actualidad existen en España nuevas iniciativas de difusión de la información geográfica patrimonial que siguen esta línea aunque, al igual que ocurría hace años, las propuestas aún siguen siendo escasas. Dado que en la actualidad casi todas las administraciones culturales autonómicas disponen de información espacial y la limitación del desarrollo tecnológico no es un obstáculo, hay que suponer que la insuficiencia de estos recursos está relacionada fundamentalmente con las cautelas que se establecen para el acceso público a esta información. 


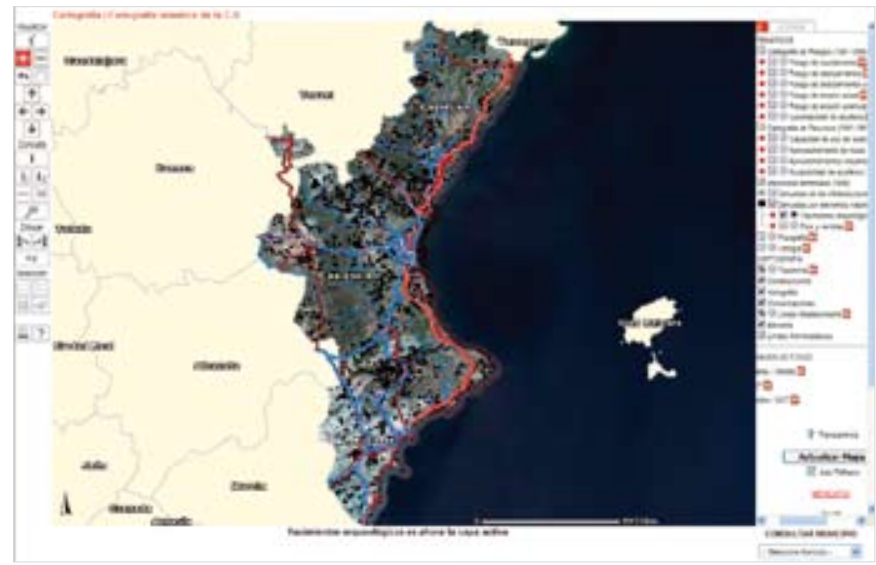

Servidor cartográfico de la Conselleria de Medio Ambiente, Agua, Urbanismo y Vivienda de la Generalitat de Valencia: orto.cma.gva.es/website/urbanismo/ viewer.htm?idioma $=c$
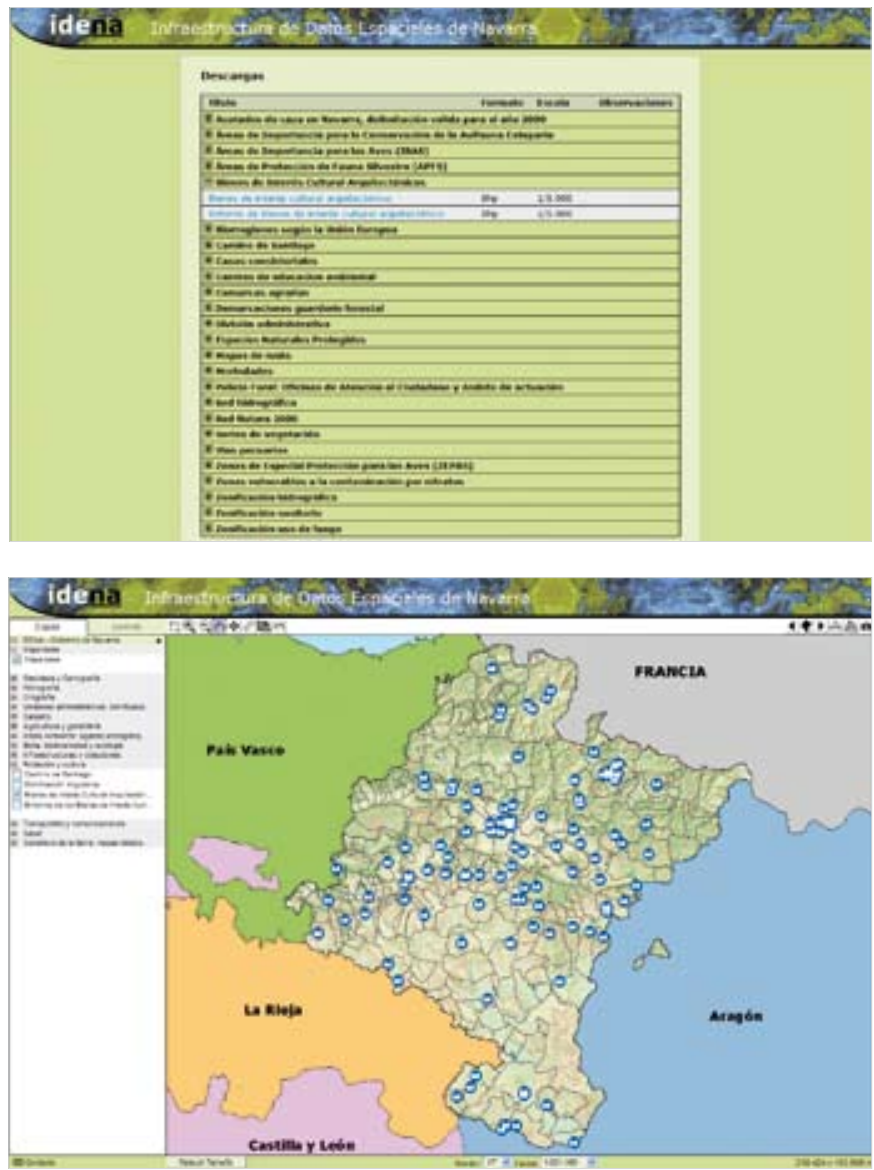

Sistema de Información Territorial de Navarra: idena.navarra.es/busquedas/ descargas.aspx (arriba); idena.navarra.es/navegar/?lang (debajo)

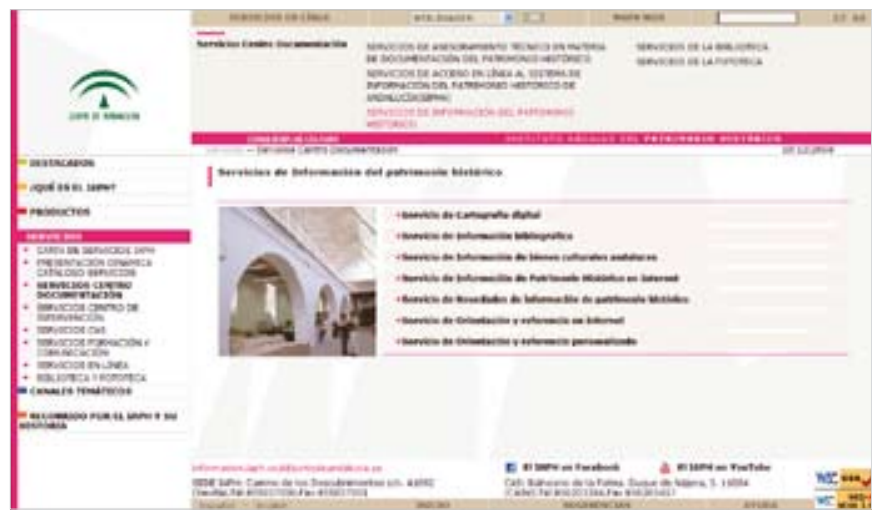

Servicios de Información del patrimonio histórico del IAPH: www.juntadeandalucia.es/cultura/iaph (navegación: Servicios - Servicios Centro Documentación)
Sin ánimo de realizar una descripción exhaustiva de todos los servicios disponibles, de entre las propuestas regionales es posible señalar una clara tendencia a la integración de éstos en las infraestructuras de datos espaciales autonómicas. En estos casos, la información cultural es accesible bien a través de su consulta en un visor cartográfico, o a través de un servicio estándar de visualización de mapas (web map service).

En este contexto, la comunidad autónoma de Murcia, a través del Sistema de Información Territorial de la Región de Murcia'; el Cabildo de Tenerife, en el Atlas digital de Tenerife ${ }^{2}$; el Gobierno de la Generalitat de Valencia, a través del servidor cartográfico de la Conselleria de Medio Ambiente, Agua, Urbanismo y Vivienda ${ }^{3}$; y la Comunidad Foral de Navarra, en el Sistema de Información Territorial de Navarra ${ }^{4}$, son ejemplos de iniciativas de integración de información patrimonial.

En ellos, siguiendo las determinaciones de la normativa europea, en la mayoría de los casos esta información hace referencia a las entidades catalogadas como Bien de Interés Cultural (BIC). Una excepción a esto lo constituyen las comunidades de Valencia y Navarra. La primera de ellas propone como información patrimonial el Inventario de Yacimientos arqueológicos. En la segunda, además de permitir la descarga en formato shape, la información temática publicada atañe únicamente a los BIC arquitectónicos.

En Andalucia, además de los contenidos normativos anteriormente señalados, el Plan Cartográfico 2009-2012, en su Apartado III, define como uno de sus objetivos, estrategias y líneas de actuación la mejora de la difusión de la información geográfica. En el marco del Sistema Cartográfico de Andalucia, "la producción de cartografía e información geográfica se concibe como un servicio destinado a satisfacer las necesidades de la sociedad en su conjunto" (PLAN, 2008).

Para desarrollar esta estrategia promueve diversas líneas de actuación, que se concretan en la disposición 65 del Apartado IV (Acceso a la información geográfica). En esta disposición con rango de norma es posible destacar, entre otras, las siguientes consideraciones:

- Necesidad de desarrollar políticas de difusión de la información geográfica con el objetivo de facilitar su uso por parte de la ciudadanía, los investigadores, las empresas y las restantes administraciones públicas.

- Necesidad de desarrollar una estrategia de difusión amplia con un uso de la mayor variedad posible de soportes y canales de distribución.

- La aplicación de condiciones restrictivas al acceso se hará de forma limitada y justificada (entre los criterios de restricción se encuentra la protección del patrimonio ambiental y cultural).

- Realización de estudios para conocer las necesidades de los usuarios y elaborar perfiles de usuarios. 
- Creación del Catálogo de Datos Espaciales como instrumento unitario de conocimiento de los datos espaciales que obren en poder de la administración pública.

En consonancia con las estrategias y disposiciones de estos documentos normativos y de planificación, la comunidad autónoma andaluza también ha puesto en marcha la Infraestructura de Datos Espaciales de Andalucia. Entre el conjunto de datos que son accesibles mediante servicios interoperables se encuentra la información del patrimonio cultural. Por su parte, el IAPH, en linea con las estrategias que promueven una mayor variedad de canales y soportes de difusión, ha desarrollado en los últimos años dos actuaciones centradas en la transferencia y difusión de la información geográfica. La primera de ellas fue la implantación de un servicio público bajo demanda para la consulta de la información geográfica. La segunda, el desarrollo de un producto para la geovisualización y consulta de esta información (PIZARRO; VILLALÓN; DÍAZ, 2008).

\section{EL SERVICIO DE CARTOGRAFÍA DIGITAL DEL IAPH}

El desarrollo del SIPHA conllevó desde un primer momento la implementación de un Sistema de Información Geográfica para gestionar y administrar la información espacial de las entidades patrimoniales. En el marco de este proyecto, desde la publicación del primer mapa digital de yacimientos arqueológicos en 1996 (AMORES et ál., 1997) hasta la actualidad, se ha ido generando un importante volumen de información geográfica que ha permitido dotar a la administración cultural de la cartografía temática del patrimonio inmueble de Andalucia.

En la medida en que se comenzaba a disponer de la información geográfica de las entidades patrimoniales, ésta fue adquiriendo una doble utilidad como instrumento para el conocimiento y la gestión del territorio. En el contexto de la labor de la Consejería de Cultura se convertía en un elemento de apoyo para el desarrollo de una adecuada politica de gestión. Por otro lado, en el marco de los proyectos con implicación territorial desarrollados por otras consejerias o empresas privadas, resultaba fundamental para facilitar el conocimiento sobre la localización y delimitación de los diferentes bienes con el objetivo de garantizar tanto la integridad de los mismos como el cumplimiento de las determinaciones que se desprendian de su situación legal.

Conscientes de ello, y con la finalidad de facilitar el acceso y el conocimiento de esta información, el IAPH definió un servicio específico bajo demanda mediante el cual se facilitaba la información geográfica del patrimonio histórico para ámbitos espaciales delimitados. Este recurso está dirigido a usuarios de carácter técnico, fundamentalmente empresas implicadas en proyectos de obras públicas, a otras administraciones, pero también a profesionales y grupos de trabajo inmersos en proyectos de investigación.
Aunque este servicio se establece de modo formal en 2005 con la publicación de la Carta de Servicios del IAPH (RESOLUCIÓN, 2005), el mismo venía prestándose desde que comenzó a estar disponible la producción cartográfica del IAPH. De igual modo, con anterioridad, el Centro de Documentación y Estudios venía proporcionando un servicio de asesoramiento interno para la Consejeria de Cultura en materia de producción cartográfica.

El Servicio de Cartografía digital forma parte de los Servicios de Información del Patrimonio Histórico del IAPH. La prestación y responsabilidad del mismo recae en el Área de Técnicas de Documentación Gráfica. Según se recoge en la Carta de Servicios se define como un "servicio para facilitar información geográfica digital en formato shape con la localización y delimitación de las entidades patrimoniales de Andalucia incluidas en el Subsistema de Información Geográfica del SIPHA. Está destinado a los investigadores y organismos que lo soliciten, previo acuerdo con el IAPH, así como a empresas y entidades que estén implicadas directamente en proyectos de obras públicas". Para la prestación de este servicio se detallan unos compromisos de calidad, que se concretan en la atención personalizada del 100\% de las demandas y en la resolución del 90\% de las peticiones, de acuerdo con los procedimientos y criterios establecidos, en el plazo máximo de 14 días. Igualmente se establecen unos indicadores para valorar este servicio, los cuales atañen al número de peticiones atendidas anualmente y al porcentaje de peticiones resueltas en un plazo inferior al máximo establecido.

El procedimiento instaurado para la petición de información geográfica conlleva, como servicio bajo demanda, el envío de una solicitud al director del IAPH. En ella deben reflejar el motivo y la justificación de la misma, adjuntando para ello cuanta información le sea solicitada. De forma paralela es necesario enviar un fichero con la delimitación del ámbito espacial objeto de demanda en formato shape o $d x f$ georreferenciado.

Una vez tramitada cada solicitud, su resolución es responsabilidad de un técnico especializado que, según su finalidad, determina y prepara la información de las entidades patrimoniales que se aportará. La respuesta se realiza a través del envío de un fichero en formato shape, que contiene la geometria y los atributos básicos de las entidades patrimoniales. Además se añade un fichero de texto en el que se describen las caracteristicas generales de la información, la fuente de origen, la fecha de actualización y el sistema de referencia de los datos espaciales, así como una descripción del modelo de datos de los atributos alfanuméricos.

El Servicio de Cartografía nace como respuesta a la necesidad de un cierto tipo de usuarios de conocer no sólo los datos básicos de las entidades patrimoniales (identificación, adscripción administrativa, descripción, adscripción crono-funcional, estado de protección, de conservación, etc.) sino que además necesita tener acceso a su dimensión espacial. 
Se trata de un servicio especializado que junto a otros, entre ellos el Servicio de Información de Bienes Culturales andaluces y el Servicio de Información del Patrimonio Histórico en Internet, complementan la oferta de los Servicios de Información del patrimonio histórico.

Desde sus inicios se ha convertido en un recurso utilizado mayoritariamente por empresas y profesionales implicados en la realización de proyectos de prevención y control ambiental, o en la redacción de documentos de planificación y ordenación territorial y urbanística, abarcando estas dos temáticas en conjunto el 95\% de las solicitudes de esta información. El 5\% restante responde a solicitudes para el desarrollo de proyectos de investigación, de difusión, etc.

La tareas de prevención ambiental son una práctica regulada por la legislación estatal y autonómica que tienen como finalidad el desarrollo de mecanismos de control sobre las actuaciones que previsiblemente puedan tener unas repercusiones negativas en el medio ambiente.

En Andalucia, los instrumentos previstos por la legislación hasta el año 2007 eran el procedimiento de Evaluación de Impacto Ambiental, el Informe Ambiental y la Calificación Ambiental. Con la aprobación de la Ley 7/2007, de 9 de julio, de Gestión Integrada de la Calidad Ambiental, se definen nuevos procedimientos: la autorización ambiental integrada, la autorización ambiental unificada, la evaluación ambiental de planes y programas, la calificación ambiental y la autorización de control de la contaminación ambiental. Exceptuando este último, el resto debe incluir en su tramitación una evaluación de impacto ambiental de las actuaciones, es decir un análisis predictivo destinado a valorar los efectos directos e indirectos de una actuación sobre el medio ambiente. Este análisis se desarrolla, por parte del titular o promotor de una actuación, a través de un estudio de impacto ambiental, documento en el que a partir de una valoración de múltiples criterios sobre la información ambiental que forma parte de un ámbito territorial delimitado se pretende conocer de forma anticipada la repercusión medioambiental de estas actividades, para de este modo actuar previamente evitando los efectos negativos.

Entre la información que se analiza en un estudio de impacto ambiental se encuentran los elementos del patrimonio histórico. Asi lo determinan la Ley 14/2007, de 26 de noviembre, del Patrimonio Histórico de Andalucia, la Ley 27/2006, de 18 de julio, por la que se regulan los derechos de acceso a la información en materia de medio ambiente, y la Ley 7/2007 de Gestión Integrada de la Calidad Ambiental.

Los promotores de cualquier actuación que como parte de su tramitación administrativa deba incluir un estudio de impacto ambiental deben recopilar la información existente o, a petición de la administración cultural, realizar una actividad arqueológica para el reconocimiento del territorio afectado.
En este sentido, el Servicio de Cartografía proporciona información espacial de las entidades patrimoniales incluidas en el ámbito territorial de cada actuación, sirviendo estos datos, junto a otros, como elementos de referencia para realizar dicho estudio y determinar el trazado o la ubicación más idónea, o bien para poder valorar la viabilidad de diferentes alternativas.

Por ello, el formato de entrega de los datos espaciales es especialmente valorado por los usuarios que, además de solicitar la geometría de las entidades, necesitan consultar los atributos básicos de las mismas.

Desde su puesta en funcionamiento, el servicio ha registrado un incremento constante del volumen de demandas, tal y como se refleja en el gráfico 1. La única excepción a esta tendencia durante el periodo analizado es el año 2008, sin que exista para ello un motivo aparente más allá de la propia dinámica del servicio o la disminución del número de afecciones territoriales. Durante el año 2009, los datos disponibles hasta el mes de noviembre apuntan de nuevo hacia un incremento de las solicitudes.

La tipología mayoritaria de usuarios de este servicio proviene de un ámbito profesional más o menos estable que desarrolla una actividad constante. Constituyen una excepción aquellas consultas que se realizan de forma puntual y aislada, siendo lo habitual en este sector que una vez conocido el recurso hagan uso del mismo de forma recurrente en la medida en que desarrollan su labor. A la fidelidad de los usuarios iniciales no dejan de sumarse otros nuevos a lo largo de estos años. Esta tendencia es especialmente reseñable si se tienen en cuenta que los dos únicos canales de conocimiento del servicio son el portal web de la Institución y la Carta de Servicios. Por tanto, teniendo en cuenta estos dos aspectos se puede concluir que el Servicio de Cartografía es un recurso activo cuya demanda sigue en constante crecimiento.

Si se analiza la distribución espacial de las solicitudes de información a lo largo del período 2006-2009, es posible observar unas proporciones constantes en este intervalo temporal. Así, el mayor porcentaje de consultas se realizan para actuaciones que se ubican en la provincia de Sevilla. En el extremo opuesto se sitúa la provincia de Almería. El resto de provincias mantiene unos porcentajes estables dentro de un rango variable entre el 9 y el $18 \%$.

Actualmente resulta dificil valorar de forma exhaustiva hasta qué punto el Servicio de Cartografía cubre las necesidades de información patrimonial que demandan los procedimientos de prevención ambiental en Andalucia. Cualquier análisis en este sentido con los datos disponibles sólo puede tener un valor estimado. Tomando el año 2007 como ejemplo, si se analizan de forma global el número de solicitudes del servicio y el número de expedientes iniciados por la Consejería de Medio Ambiente de la Junta de Andalucía, la valoración resulta desigual. Si se atiende únicamente a los expedientes de evaluación de impacto am- 

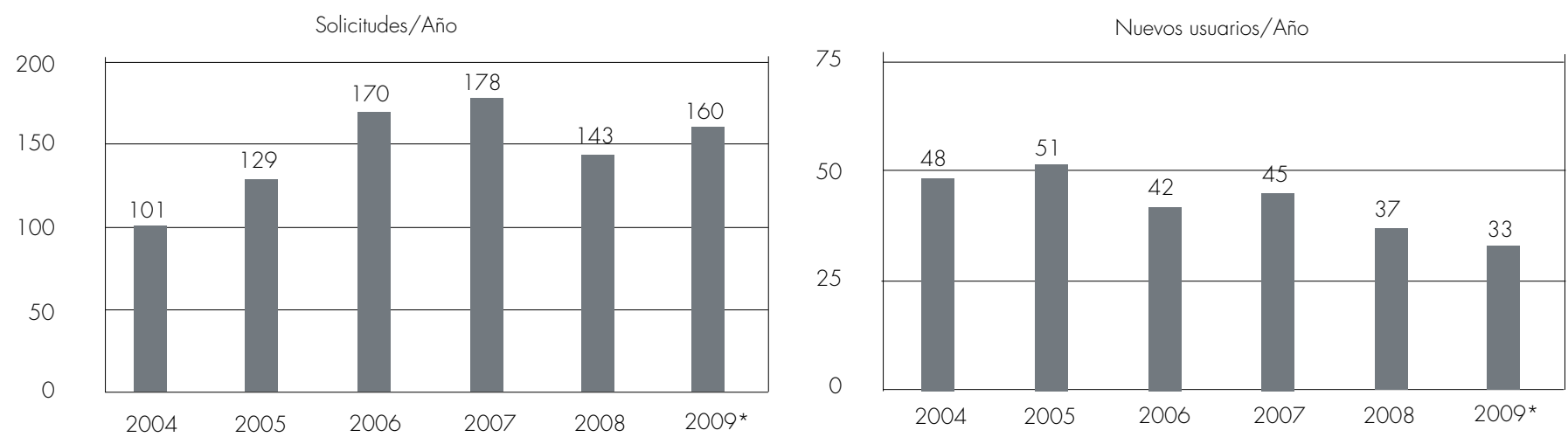

* Datos estimados

Gráficos 1 y 2. Servicio de Cartografía. Solicitudes/año y Nuevos usuarios/año. Fuente: elaboración propia

\% Distribución provincial de la información solicitada 2006

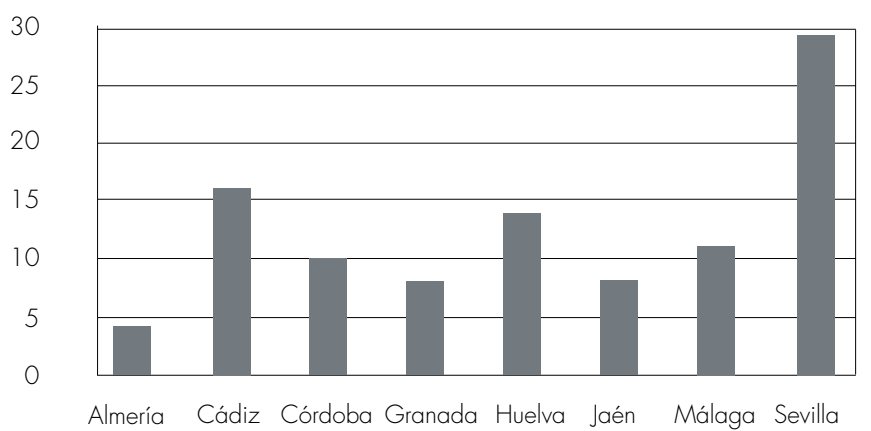

\% Distribución provincial de la información solicitada 2008

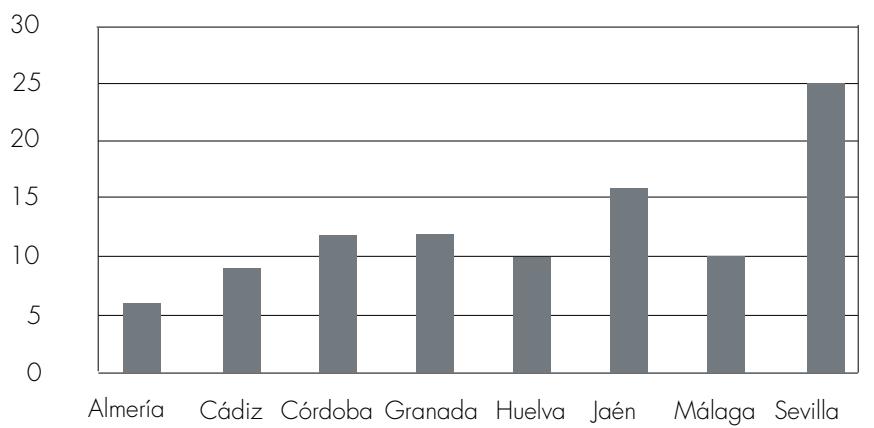

\% Distribución provincial de la información solicitada 2007

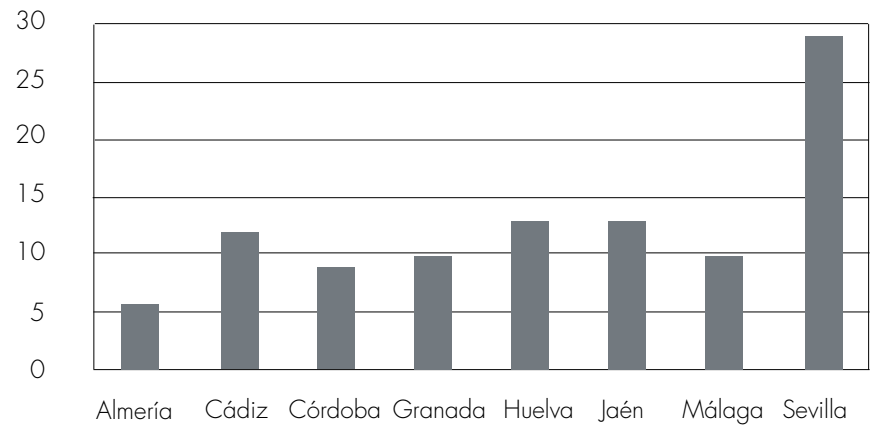

\% Distribución provincial de la información solicitada 2009

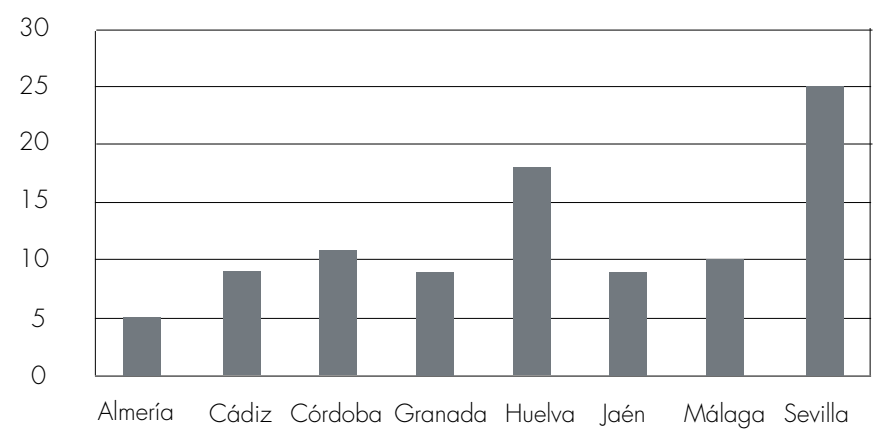

Gráficos 3, 4, 5 y 6. Servicio de Cartografía. Distribución provincial de la información solicitada. Años 2006-2009, respectivamente. Fuente: elaboración propia

Prevención Ambiental 2007

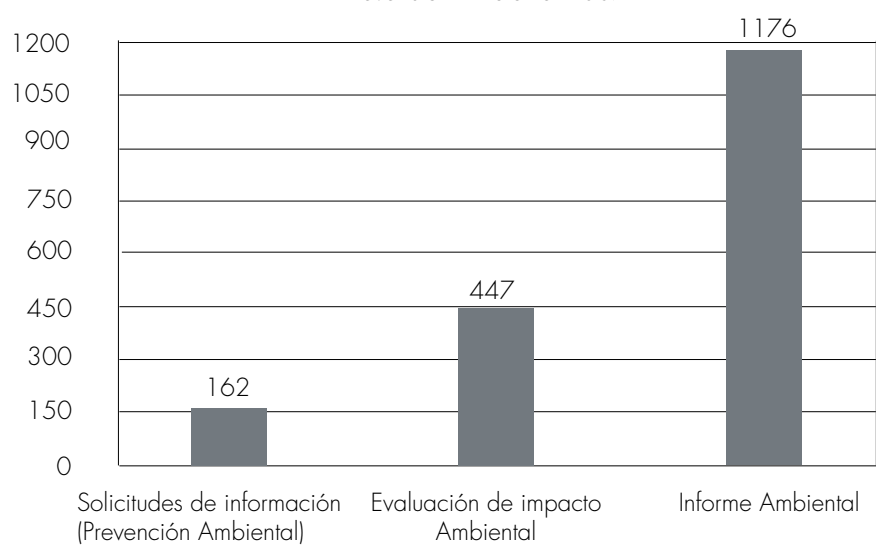

Gráfico 7. Prevención ambiental 2007. Fuente: elaboración propia a partir de datos de la Consejería de Medio Ambiente de la Junta de Andalucía
Desde sus inicios, el Servicio de Cartografía se ha convertido en un recurso utilizado mayoritariamente por empresas y profesionales implicados en proyectos de prevención y control ambiental, o en la redacción de documentos de planificación y ordenación territorial y urbanística 


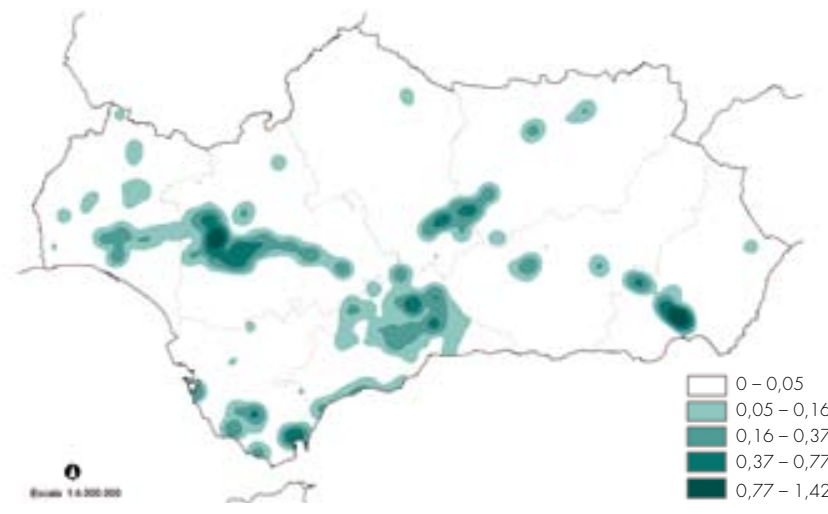

Distribución de la información proporcionada. Año 2006.

Fuente: elaboración propia

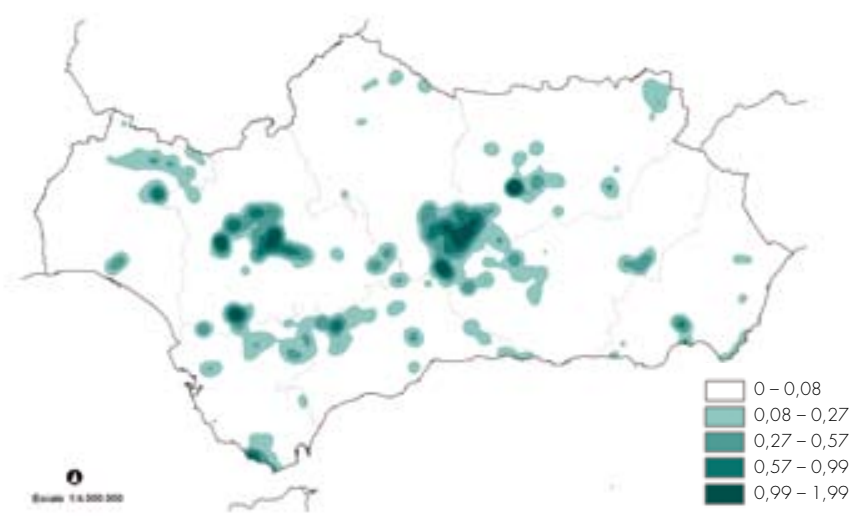

Distribución de la información proporcionada. Año 2008

Fuente: elaboración propia

biental (procedimiento para las actuaciones contempladas en el Anexo I de la legislación en vigor en esa fecha) se puede estimar que el servicio cubre un 35\% del total. Sin embargo, si a estos expedientes se añaden los de informe ambiental (procedimiento para las actuaciones del Anexo II), el porcentaje desciende al $14 \%$. De todos modos hay que tomar estos resultados con precaución, ya que para realizar esta evaluación de forma correcta habria que efectuar un estudio detallado de cada actuación. Sin embargo, pueden ser un punto de partida para realizar una valoración en este sentido, especialmente si se tiene en cuenta que la mayor parte de las solicitudes de información sobre estudios de impacto ambiental del Servicio de Cartografía está vinculada al primer procedimiento.

En todo caso, durante estos años el servicio viene prestando una labor que repercute claramente en la prevención de afecciones sobre el patrimonio, como puede deducirse del análisis de los mapas que muestran la densidad y distribución espacial de la información patrimonial proporcionada para diferentes proyectos.

\section{CONCLUSIONES}

A lo largo de la última década se han desarrollado diferentes disposiciones, normativas e instrumentos de planificación que

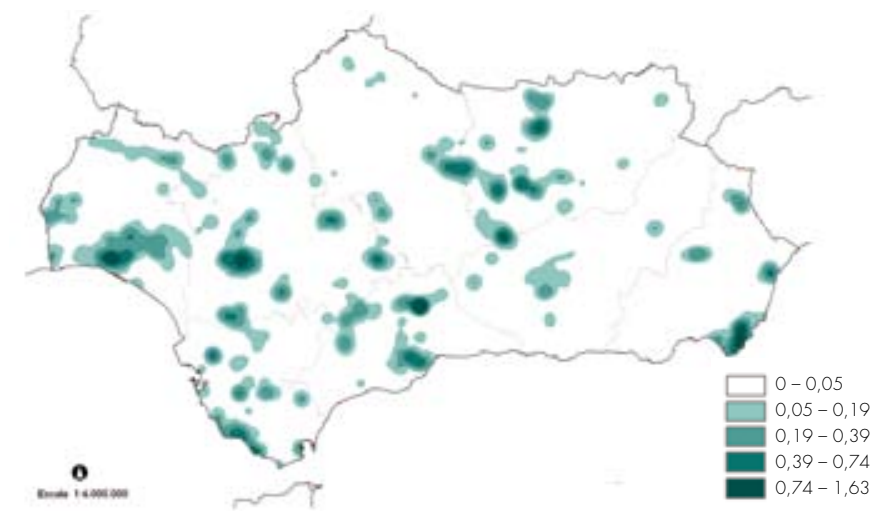

Distribución de la información proporcionada. Año 2007. Fuente: elaboración propia

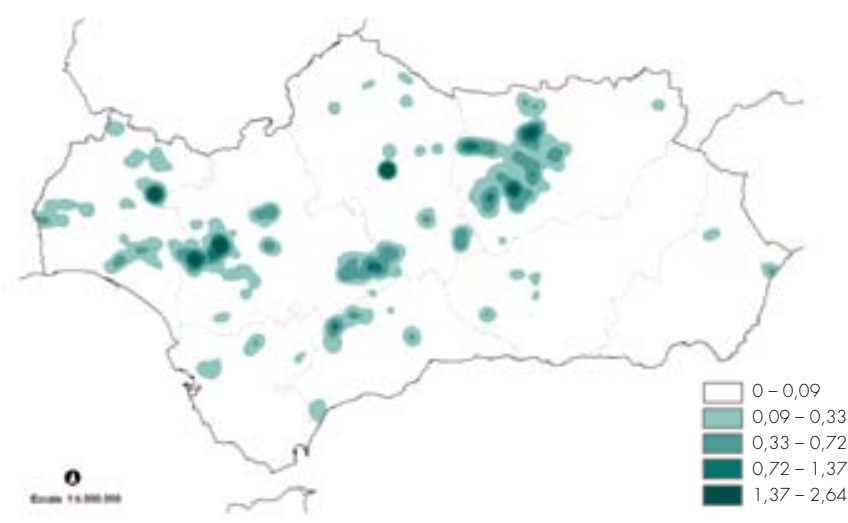

Distribución de la información proporcionada. Año 2009.

Fuente: elaboración propia

inciden en la relevancia del papel estratégico de la información geográfica en las tareas de la administración. Con este objetivo, estos documentos tratan de regular los procesos de producción y, especialmente, las estrategias de difusión para mejorar las capacidades de acceso a la misma.

De forma paralela, y en consonancia con estas iniciativas, durante este periodo se ha producido una evolución de las infraestructuras y tecnologias de la información geográfica, lo cual ha permitido el surgimiento de nuevas vías para facilitar el acceso y distribución de la información espacial, ya sea a través de servicios estándar de visualización de mapas o de servicios de descargas de datos.

Las políticas de difusión de las administraciones públicas en materia de información geográfica están tendiendo a seguir esta línea y, a través de la puesta en marcha de las infraestructuras de datos espaciales, comienzan a poner a disposición de diferentes usuarios la información territorial que gestionan. En el ámbito de la administración cultural, las limitaciones a su acceso público están vinculadas a criterios relacionados con su protección y preservación, especialmente en el caso de los sitios arqueológicos. Sin embargo, justamente para protegerlo es fundamental conocerlo. La Directiva Inspire incluye, entre el conjunto de datos espaciales cuya producción y disponibilidad han de quedar aseguradas, a los bienes de interés cultural. En esta línea, en 
Andalucia, al igual que en otras comunidades, las estrategias de la Consejería de Cultura se dirigen en este sentido.

Sin embargo, las tareas de prevención y planificación ambiental y territorial no pueden limitarse al conocimiento parcial de la realidad patrimonial, sino que han de contemplarla en su globalidad. Por ello es fundamental la existencia de diferentes alternativas en cuanto a soportes y canales de distribución, como queda recogido en el documento de Planificación de la actividad cartográfica en Andalucia.

Desde su puesta en marcha, el Servicio de Cartografía digital del IAPH se ha consolidado como un servicio especializado para la transferencia de la información espacial del patrimonio cultural andaluz. Su integración en la Carta de Servicios asegura que la prestación del mismo se ofrece bajo una oferta pública y en el contexto de los procesos generales de mejora de la calidad de los servicios de la administración andaluza.

Durante estos años, los diferentes usuarios de este servicio valoran fundamentalmente el acceso y el contenido de la información que proporciona, así como las orientaciones técnicas y la rápida diligencia de sus solicitudes. Por el contrario, el principal inconveniente que manifiestan se deriva del procedimiento establecido para la tramitación de un servicio bajo demanda.

De cara al futuro, el IAPH sigue trabajando en el desarrollo de una serie de iniciativas para mejorar tanto los procedimientos de accesibilidad como para diversificar la información proporcionada, adecuándola a las necesidades de todos los usuarios.

\section{Notas}

${ }^{1}$ Sistema de Información Territorial de la Región de Murcia (SITMUR): la Dirección General de Ordenación del Territorio ofrece la posibilidad de consultar la información del patrimonio cultural a través de un visor (http://massotti.carm.es/website/ str/viewer) o a través de un servicio WMS ( http://massotti.carm.es/wmsconnector/ com.esri.wms.Esrimap/wms?).

${ }^{2}$ Atlas Digital de Tenerife (Geoportal de Tenerife): el Cabildo de la Isla de Tenerife permite la consulta de la capa de Bienes de Interés Cultural a través de un visor (http://atlastenerife.es/TelDE2/Visor.do) o mediante acceso al servicio WMS (http:// atlastenerife.es/MAPSERVER/cgi-bin/mapserv_VIS-00000211.exe).

${ }^{3}$ Servidor cartográfico de la Conselleria de Medio Ambiente, Agua, Urbanismo y Vivienda: la Generalitat Valenciana permite la consulta del Inventario de Yacimientos Arqueológicos a través del visor del servidor cartográfico de la Conselleria de Medio Ambiente, Agua, Urbanismo y Vivienda (http://orto.cma.gva.es/website/ urbanismo/viewer.htm?idioma $=c$ ) o a través de un servicio WMS (http://orto.cth. gva.es/wmsconnector/com.esri.wms.Esrimap/wms_urbanismo_tematicos?).

${ }^{4}$ Sistema de Información Territorial de Navarra (SITNA): la Infraestructura de Datos Espaciales de Navarra ofrece la consulta de los Bienes de Interés Culturales arquitectónicos y su entorno a través de un visor (http://idena.navarra.es/ navegar/?lang= ) y de un servicio de descarga en formato shp (http://idena.navarra.es/busquedas/descargas.aspx).

\section{Bibliografía}

AMORES, F.; FERNÁNDEZ, S.; GARCÍA, L.; HURTADO, V.; LADRÓN DE GUEVARA, C.; MÁROUEZ, H.; RODRÍGUEZ-BOBADA, M. C. (1997) Planteamientos y primeros resultados de la transferencia a soporte $\mathrm{SIG}$ del inventario de Yacimientos Arqueológicos de Andalucia. PH Boletín del IAPH, no 18, 1997, pp. 124-143

BLANCO MURIEL, A.; LIMÓN RODRÍGUEZ, S; MADRID DÍAZ, M. V. (2007) La difusión de la información. En El sistema de Información del Patrimonio Histórico de Andalucia (SIPHA). Sevilla: Consejeria de Cultura, 2007, pp. 205-217 (PH Cuadernos; 20)

BLANCO MURIEL, A.; ORTEGA VAQUERO, I. (2005) La carta de servicios del Instituto Andaluz del Patrimonio Histórico. PH Boletín del IAPH, no 53, 2005, pp. 78-83

DIRECTIVA 2007/2/CE, del Parlamento Europeo y del Consejo de 14 de marzo de 2007 por la que se establece una infraestructura de información espacial en la Comunidad Europea (Inspire). Diario Oficial de la Unión Europea, L 108 1-14

ESTUDIO de Implantación de los Sistemas de Información Territorial en la Administración Estatal y Autonómica. Tomo I (2004) (en línea). Murcia: Consejería de Industria y Medio Ambiente, Dirección General de Ordenación del Territorio y Costas <www.sitmurcia.es/estudio/tomo1.pdf> [consulta 25-11-2009] ESTUDIO de Implantación de los Sistemas de Información Territorial en la Administración Estatal y Autonómica. Tomo II (2004) (en linea). Murcia: Consejeria de Industria y Medio Ambiente, Dirección General de Ordenación del Territorio y Costas <www.sitmurcia.es/estudio/tomo2.pdf > [consulta 25-11-2009] LADRÓN DE GUEVARA, C.; MUÑOZ, V. (2007) Introducción. En El sistema de Información del Patrimonio Histórico de Andalucía (SIPHA). Sevilla: Consejería de Cultura, 2007, pp. 10-12 (PH Cuadernos; 20)

LEY 14/2007, de 26 de noviembre, del Patrimonio Histórico de Andalucia. Boletín Oficial de la Junta de Andalucía, núm. 248, 2007, pp. 6-28

LEY 7/2007, de Gestión Integrada de la Calidad Ambiental. Boletín Oficial del Estado, núm. 190, 2007, pp. 34118-34169

LEY 27/2006, de 18 de julio, por la que se regulan los derechos de acceso a la información, de participación pública y de acceso a la justicia en materia de medio ambiente (incorpora las Directivas 2003/4/CE y 2003/35/CE). Boletín Oficial del Estado, núm. 171, 2006, pp. 27109-27123

MUÑOZ CRUZ, V.; ORTEGA VAQUERO, I. (2001) La base de datos del Patrimonio Inmueble de Andalucia en Internet: BDI-PHA. PH Boletín del IAPH, no 36, 2001, pp. 238-243

PIZARRO MORENO, C.; VILLALÓN TORRES, D.; DÍAZ IGLESIAS, J.M. (2008) EI localizador cartográfico del Patrimonio Cultural andaluz. PH Boletín del IAPH, n' 67, 2008, pp. 16-29

PLAN Cartográfico de Andalucía 2009-2012: Acuerdo del Consejo de Gobierno de 16 de septiembre de 2008 (2008). Sevilla: Consejeria de Vivienda y Ordenación del Territorio, 2008

RESOLUCIÓN de 17 de enero de 2005, de la Dirección General de Bienes Culturales, por la que se aprueba la Carta de Servicios del Instituto Andaluz del Patrimonio Histórico. Boletín Oficial de la Junta de Andalucía, núm. 33, 2005, pp. $15-23$

RODRÍGUEZ, A. F.; MAS, S.; ABAD, P.; ALONSO, J. A.; AYUSO, J. E.; SÁNCHEZ, A.; VILCHES, L. M. (2007) Una nueva etapa: hacia la IDE 2.0. En IV Jornadas Técnicas de la IDE de España (JIDEE 07). Santiago de Compostela: Asociación Galega de Cartografia e Sistemas de Información Xeográfica, 2007

SÁNCHEZ BENAVIDES, F.; ORTEGA VAQUERO, I.; DÍAZ IGLESIAS, J. M. (1999) Las bases de datos del Patrimonio Histórico andaluz en el servidor web del IAPH. $1^{\text {a }}$ Fase: ARQUEOS. PH Boletín del IAPH, nº 29, 1999, pp. 216-220 